\title{
Insects of forensic importance associated to cadaveric decomposition in a rural area of the Andean Amazon, Caquetá, Colombia
}

\author{
Yardany RAMOS-PASTRANA ${ }^{1,2^{*}}$, Yenny VIRGÜEZ-DÍAZ ${ }^{1}$, Marta WOLFF ${ }^{2}$ \\ Universidad de la Amazonia, Laboratório de Entomología, Grupo de Investigación en Entomología -GIEUA-, Florencia, Caquetá, Colombia. \\ 2 Universidad de Antioquia, Instituto de Biología, Grupo de Entomología -GEAU-, Medellín, Colombia. \\ * Corresponding author: ya.ramos@udla.edu.co
}

\begin{abstract}
Forensic entomology is a frequently used tool to estimate the time interval between death and the discovery of the corpse. Succession of arthropods associated with cadaveric decomposition was monitored in a rural area of the Municipality of Florencia, Department of Caquetá, Colombia. Three pigs (Sus scrofa) were used as study models. Insect sampling, and monitoring of carcasses and environmental conditions were carried out every five hours. The total time from death to skeletonization was of 545 hours (22.7 days). A total of 30833 insect individuals were collected. Specimens were distributed in nine orders, 46 families, 95 genera and 106 species. Diptera was the most abundant, with 23215 individuals (75.3\%), followed by Coleoptera, with 3711 individuals (12\%), and Hymenoptera, with 3154 individuals (10.2\%). Immature stages of Cochliomyia macellaria, Chrysomya albiceps, Hemilucilia semidiaphana and Ophyra aenescens were the main species involved in tissue consumption and acceleration of the decomposition process. Due to the presence of ants Cheliomyrmex sp., Camponotus sp. and Dinoponera sp., and coleopterans Hister sp., Acylophorus sp. and Philonthus spp., it was not possible to obtain sufficient Diptera egg masses for rearing the colonizing species. These results can be used as a standard to determine the postmortem interval in criminal investigations in the rural area of the Andean Amazon, Caquetá, Colombia.
\end{abstract}

KEYWORDS: cadaveric decomposition, carrion, forensic entomology, Colombian Amazon

\section{Insetos de importância forense associados à decomposição cadavérica em uma área rural na Amazônia Andina, Caquetá, Colômbia}

\section{RESUMO}

A entomologia forense é uma ferramenta frequentemente utilizada para estimar o intervalo de tempo entre a morte e a descoberta do corpo. $\mathrm{Na}$ área rural do município de Florencia, Caquetá, foi monitorada a sucessão de artrópodes associados à decomposição cadavérica, utilizando como modelo de estudo os cadáveres de três porcos (Sus scrofa). A amostragem de insetos e coleta de dados ambientais foram realizadas a cada cinco horas. A duração total do processo desde a morte até a esqueletização foi de 545 de horas (22,7 dias). Foram coletados 30833 espécimes de insetos, distribuídos em nove ordens, 46 famílias, 95 gêneros e 106 espécies. Diptera foi o grupo mais representativo, com 23215 indivíduos (75,3\%), seguido de Coleoptera, com 3711 indivíduos (12\%) e Hymenoptera, com 3154 indivíduos (10,2\%). Os estágios imaturos de Cochliomyia macellaria, Chrysomya albiceps, Hemilucilia semidiaphana e Ophyra aenescens foram as principais espécies envolvidas no consumo de tecidos e na aceleração do processo de decomposição. Devido à presença de formigas Cheliomyrmex sp., Camponotus sp. e Dinoponera sp. e de besouros Hister sp., Acylophorus sp. e Philonthus spp., não foi possível obter massas de ovos de Diptera suficientes para a criação da espécie colonizadora. Nosso estudo fornece subsídios para determinar o intervalo post-mortem em investigaçóes policiais e promover a entomologia forense em uma área rural da Amazônia Andina na Colômbia.

PALAVRAS-CHAVE: decomposição cadavérica, carcaça, entomologia forense, Amazônia colombiana 


\section{INTRODUCTION}

Forensic entomology is the science that studies insects and other arthropods associated to corpse decomposition, and is used as a tool to determine the place and cause of suspicious deaths (Anderson and VanLaerhoven 1996; Magaña 2001). It is often used to estimate the time interval between death and the discovery of the carcass, a period known as Post-mortem Interval (PMI). Frequently, when remains are found weeks, months or even longer after death, entomological evidence is the only method available to reliably determine the PMI (Anderson and VanLaerhoven 1996).

Insects colonize corpses in a predictable order, with some species attracted by fresh corpses, while others are attracted by different stages of putrefaction. Each group of insects feeding on a corpse, and benefiting from particular characteristics of the tissue at that time, further modifies this resource (Hobischak et al. 2006; Anderson 2010). What is not attractive for a particular species may be appropriate for another, and this is known as the facilitation model (Payne 1965; Hobischak et al. 2006; Anderson 2010).

PMI estimation depends, to some extent, on the composition and dynamics of the local necrophagous arthropod communities (Pujol-Luz et al. 2006). Species of Diptera colonize decomposing bodies in forest, rural and urban habitats, thus, the diversity and natural history of the local flies are important elements for PMI estimation. Furthermore, since some Diptera species have specific habitats and distribution in different environments, this group of organisms can be a good indicator of corpse relocation (Catts and Haskell 1990).

Monitoring of insects associated to cadaveric decomposition in rural environments has been studied in countries located in temperate zones such as Australia (Archer 2003), Belgium (Dekeirsschieter et al. 2013), USA (Tullis and Goff 1987) and Canada (Hobischak et al. 2006). In the Neotropics, studies have been done in Argentina (Centeno et al. 2002) and Brazil (Carvalho et al. 2004; Cruz and Vasconcelos 2006). In general terms, when independent studies (rural and/or urban) are compared, it is clear that the time between decomposition stages, the species composition, the pioneer species and the colonization times differed depending on the environment where the corpse was.

In Colombia, studies analyzing cadaveric decomposition in rural areas, with pigs (Sus scrofa Linnaeus, 1758) as study models, were done in the Municipality of Consacá, Department of Narińo, at 1,720 masl (Salazar-Ortega 2008) and in the Municipality of Pereira, Department of Risaralda, at 1,550 masl (Grisales et al. 2010), and there is one study (RamosPastrana and Wolff 2011) focused on cadaveric decomposition under sunny and shady conditions in a semi-rural area of the Colombian Amazonian Piedmont. With only three studies published on characterization of necrophagous insects in rural areas of Colombia, it becomes evident that more studies on this topic are needed. Thus, the objective of this study was to describe, characterize and monitor the entomofauna associated to the decomposition of three pigs exposed to environmental conditions in a rural area of the Amazonian Piedmont.

\section{MATERIALS AND METHODS}

This study was conducted at the César Augusto Estrada Gonzales "Macagual” Research Center, Universidad de la Amazonia, located in a rural area of the Municipality of

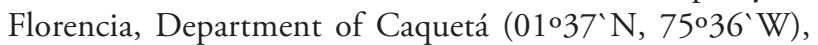
Colombia, at 280 masl. Annual mean rainfall is $3,600 \mathrm{~mm}$, with an annual average temperature of $27{ }^{\circ} \mathrm{C}$ and annual mean relative humidity of $85 \%$ (IGAC 2010). The region is characterized by transition landscape between the Amazonian Piedmont and the lower Amazonian floodplains. The study area features small hills and alluvial terraces, with landscape transitions from flat to undulated, and abrupt (IGAC 2010). The vegetation is composed of Anaxagorea spp., Xylopia spp. (Annonaceae); Virola spp., Iryanthera spp. (Myristicaceae); Pseudolmedia laevis (Ruiz \& Pav.) J.F. Macbr., Perebea spp. (Moraceae); Inga spp., Ormosia sp., Enterolobium spp., Parkia sp., Tachigali sp. (Fabaceae); Miconia spp. (Melastomataceae); Protium spp.; Crepidospernum rhoifolium (Benth.) Triana \& Planch. (Cimaz 2007). According to Holdridge (1996), this zone corresponds to a tropical rainforest (bh-T).

Three domestic pigs (Sus scrofa domestica), weighing approximately $9 \mathrm{~kg}$ each, were used as study models. Pigs were killed at the study site by a licensed veterinarian, endorsed by the ethics committee of the Universidad de La Amazonia. The pigs were administered a lethal Eutanex intracardiac injection of $3 \mathrm{ml}$ on December $21^{\text {st }}, 2010$, at approximately $10 \mathrm{am}$. Immediately after death, each pig was placed in an individual metal cage $(100 \mathrm{~cm} \times 50 \mathrm{~cm} \times 60 \mathrm{~cm})$, made of a $2 \mathrm{~cm} \times 2 \mathrm{~cm}$ wire mesh. This allowed access for insects and other arthropods, while preventing the remains from being disturbed by vertebrate scavengers. The three cages were placed $100 \mathrm{~m}$ apart from each other in patches of secondary vegetation.

Pitfall traps (25) were installed around each carcass, approximately $1 \mathrm{~m}$ away from the cage. Traps contained $75 \%$ ethanol to capture adult and/or immature arthropods approaching or moving away from the carcass. From the time of death until the remains phase, sampling was carried out every five hours without day-night interruption. Photographs were taken and physical changes were recorded. Physical changes were based on carcass weight, determined with a digital scale, and rectal carcass temperature (Elan digital thermometer). We also monitored the environmental temperature and relative humidity (thermo-hygrometer digital Thermo).

Sampling of adult flying insects was done using entomological nets. Non-flying adult and immature individuals found under and/or around the carcass were collected using tweezers and fine tip brushes, following the methodology proposed by 
Haskell (1990). Adult insects were killed in a killing jar with ethyl acetate. Some of these were separated by morphospecies and mounted on entomological pins for posterior taxonomic identification, and the remaining adults, as well as larvae, pupae and puparia, were fixed in $75 \%$ ethanol.

The individuals collected were taxonomically identified to species level, when possible, using the keys proposed by Smith (1986), Navarrete-Heredia et al. (2002), Fernández (2003), Fernández and Sharkey (2006), Flórez and Wolff (2009), Brown et al. (2009), Brown et al. (2010), and Carvalho et al. (2012). Diptera larvae were cleared with $\mathrm{KOH}$ for taxonomic identification, as this aids in the observation of microscopic structures such as the cephalopharyngeal skeleton, anterior and posterior spiracles, and size and distribution of tubercle spines (Greenberg and Szyska 1984). The specimens were deposited in the Entomological Collection of the Universidad de la Amazonia (CEUAM).

Insect succession patterns were evaluated during the decomposition of the three corpses. Each phase was delimited based on physical change of the carcasses (rectal temperature and weight loss) and was related to the presence, development stage and abundance of its associated insects, and to environmental variables, according to the criteria established by Anderson and VanLaerhoven (1996) and Ramos-Pastrana et al. (2014). A succession table and an occurrence matrix were generated using presence-absence data. The ecological category of sampled insetc species was determined according to Smith (1986) and Magaña (2001).

\section{RESULTS}

A total of 30833 individual insects, both immatures and adults, distributed in nine orders, 46 families, 95 genera and 106 species, were recorded in this study (Table 1). Overall, the most abundant order was Diptera, with 23215 individuals (75.3\% of the total) followed by Coleoptera $(3711,12 \%)$ and Hymenoptera (3154, 10.2\%). Within Diptera, the most abundant families were Calliphoridae (10449, 44.9\%), Muscidae (10011, 43.1\%) and Sarcophagidae (1492, 6.4\%) of the total of Diptera (Tables 2 and 3). The most abundant families of Coleoptera were Histeridae (1974, 53.1\%), Staphylinidae (1229, 33.1\%) and Scarabaeidae $(439,11.8 \%)$ of the total of Coleoptera (Tables 2 and 3). In Hymenoptera, the family Formicidae dominated with 3012 individuals (95.5\%) of the total of Hymenoptera.

\section{Decaying stages and entomofauna behavior}

The average decomposition time was 545 hours (22.7 days) and five decaying stages of the carcasses were defined (fresh, bloated, active decay, advanced decay, and remains) based on the physical change of the carcasses, their temperature and weight loss.

Stage 1 - fresh carcass (hour 0 to 15) - This stage started at the moment of death and lasted until the visible onset of carcass bloating. It was characterized by lividity, dehydration and rigor mortis, mainly in the extremities of the corpse. Only adult insects were observed at this stage, mainly ants, and, to a lesser extent, flies of the families Calliphoridae, Muscidae and Sarcophagidae; some coleopterans and spiders were also present (Tables 1 and 2, Supplementary Material, Table S1). The predatory activity of the Formicidae species was responsible for the observed decrease in dipteran eggs. Rectal carcass temperature decreased sharply from $27.33^{\circ} \mathrm{C}$ to $22^{\circ} \mathrm{C}$, environmental temperature oscillated between $23.67^{\circ} \mathrm{C}$ and $27.33^{\circ} \mathrm{C}$, and relative humidity ranged between $79 \%$ and $86.33 \%$ (Figure 1a). During this stage, carcass weight loss was of $5.98 \%$ (Figure 1b).

Stage 2 - bloated carcass (hour 20 to 80) - This desintegration phase started when the swelling of the carcass was evident by the gases generated by anaerobic bacteria decomposition. It was characterized by the spherical shape of the carcass, the fluid outlet by mouth and nose, the blue-green coloration in the upper part of the carcass and the purple coloration in the area in contact with the soil. Concerning the entomofauna, the first immature dipterans were detected, belonging mainly to Calliphoridae (Tables 1 and 3, Supplementary Material, Table S1), while in the adult stage ants sill dominated, mainly Camponotus sp., followed by Coleoptera and Diptera (Tables 1 and 2, Supplementary Material, Table S1). Rectal carcass temperature fluctuated between 22 and $24.33{ }^{\circ} \mathrm{C}$, while environmental temperature oscillated between 23.33 and $36{ }^{\circ} \mathrm{C}$, and relative humidity between $69 \%$ and $98 \%$ (Figure 1a). Weight loss was of $9.63 \%$, for an accumulated total of $15.61 \%$ (Figure 1b).

Stage 3 - active decay (hour 85 to 115) - This phase started with the loss of volume, which allows gases to escape, and the subsequent presence of strong putrefaction odors. In addition, it was characterized by a humid carcass and the rupture of the skin, complete consumption of the head tissues, and shedding of the skin from the abdomen and extremities. The carcass started to lose its original shape, while still preserving its muscular and epithelial tissues. The scavenger entomofauna was represented by immature Diptera of $H$. semidiaphana (LIII), O. aenescens (LII, LIII) (Muscidae) and nymphs of Blattidae (Tables 1 and 3, Supplementary Material, Table S1). Adults were represented by Diptera, mainly Calliphoridae, Muscidae and Sarcophagidae; Coleoptera, mainly Philonthus sp1. and Hymenoptera (Formicidae), mainly Camponotus sp. (Tables 1 and 2, Supplementary Material, Table S1). Rectal carcass temperature fluctuated between 22 and $24.33^{\circ} \mathrm{C}$; environmental temperature between 23.33 and $29^{\circ} \mathrm{C}$, and relative humidity between $64.33 \%$ and $80 \%$ (Figure 1a). Carcass weight loss was of $9.17 \%$, for an accumulated total of $24.8 \%$ (Figure 1b).

Stage 4 - advanced decay (hour 120 to 170) - This phase started with considerable tissue loss, only patches of skin left preserved, and was characterized by reduced odors and adult insect activity of Diptera. At the end of this stage, the carcass was almost completely disarticulated, with only fats and 
Table 1. Succession of immature (I) and adult (A) insects in different stages of cadaveric decomposition (fresh, bloated, active, advanced and remains) of pigs in a rural area of the Andean Amazon, Caquetá, Colombia. Numbers below the decomposition stage indicate the duration of the stage in hours from 0 to 545 . CAT indicates the ecological niche of the species (N: Necrophagous; PP: Predator or Parasite; O: Omnivore; A: Accidental).

\begin{tabular}{|c|c|c|c|c|c|c|c|c|c|c|c|}
\hline \multirow{3}{*}{ Species } & \multirow{3}{*}{ CAT } & \multicolumn{10}{|c|}{ Stages of descomposition - Hours } \\
\hline & & \multicolumn{2}{|c|}{ Fresh $0-15$} & \multicolumn{2}{|c|}{ Bloated 20-80 } & \multicolumn{2}{|c|}{ Active $85-115$} & \multicolumn{2}{|c|}{ Advanced 120-170 } & \multicolumn{2}{|c|}{ Remains $175-545$} \\
\hline & & I & A & I & A & I & A & I & A & 1 & A \\
\hline \multicolumn{12}{|l|}{ Order DIPTERA } \\
\hline \multicolumn{12}{|l|}{ Family Calliphoridae } \\
\hline Cochliomyia macellaria & $\mathrm{N}$ & & & $x$ & $x$ & & $x$ & $x$ & $x$ & $x$ & $x$ \\
\hline Chrysomya albiceps & $N, P P$ & & & & $x$ & & $x$ & $x$ & $x$ & $x$ & $x$ \\
\hline Hemilucilia semidiaphana & N & & & $x$ & $x$ & $x$ & $x$ & $x$ & $x$ & $x$ & $x$ \\
\hline Paralucilia paraensis & N & & & & $x$ & & $x$ & & $x$ & & $x$ \\
\hline Choroprocta idioidea & $\mathrm{N}$ & & & & $x$ & & $x$ & & & & \\
\hline Lucilia eximia & $\mathrm{N}$ & & $x$ & & $x$ & & & & & & $x$ \\
\hline \multicolumn{12}{|l|}{ Family Sarcophagidae } \\
\hline Microcerella sp. & $\mathrm{N}$ & & & & $x$ & & $x$ & & & & $x$ \\
\hline Peckia sp. & N & & & & $x$ & & & & $x$ & & $x$ \\
\hline Tricharaea sp. & N & & $x$ & & $x$ & & $x$ & & $x$ & & $x$ \\
\hline Blaesoxipha sp. & N & & & & $x$ & & $x$ & & & & $x$ \\
\hline Sarcodexia sp1. & N & & & & $x$ & & $x$ & & $x$ & & $x$ \\
\hline Sarcodexia sp2. & N & & & & & & & & $x$ & & \\
\hline Sarcophaga sp. & N & & & & $x$ & & & & & & \\
\hline Oxysarcodexia sp. & N & & & & $x$ & & $x$ & & $x$ & & $x$ \\
\hline Boettcheria sp. & N & & & & $x$ & & & & & & \\
\hline Unidentified species. & $\mathrm{N}$ & & & $x$ & & $x$ & & $x$ & & & \\
\hline \multicolumn{12}{|l|}{ Family Muscidae } \\
\hline Ophyra aenescens & N & & & & $x$ & $x$ & $x$ & $x$ & $x$ & $x$ & $x$ \\
\hline Biopyrellia bipuncta & N & & $x$ & & $x$ & & $x$ & & $x$ & & $x$ \\
\hline Trichomorellia flavipalpis & N & & & & $x$ & & $x$ & & $x$ & & $x$ \\
\hline \multicolumn{12}{|l|}{ Family Piophilidae } \\
\hline Piophila sp. & $\mathrm{N}$ & & & & $x$ & & & & $x$ & & $x$ \\
\hline \multicolumn{12}{|l|}{ Family Syrphidae } \\
\hline Copestylum sp1. & PP & & & & $x$ & & & & $x$ & & $x$ \\
\hline Copestylum sp2. & PP & & & & $x$ & & & & $x$ & & $x$ \\
\hline Copestylum sp3. & PP & & & & $x$ & & & & $x$ & & $x$ \\
\hline Copestylum sp4. & PP & & & & & & $x$ & & & & $x$ \\
\hline Ornidia sp. & PP & & & & $x$ & & & & & & $x$ \\
\hline Salpingogaster sp. & PP & & & & & & & & & & $x$ \\
\hline \multicolumn{12}{|l|}{ Family Tabanidae } \\
\hline Chrysops sp. & A & & & & & & $x$ & & & & $x$ \\
\hline Diachlorus sp. & A & & & & $x$ & & & & & & \\
\hline Tabanus sp. & A & & & & & & & & & & $x$ \\
\hline Family Tachinidae & & & & & & & & & & & \\
\hline Specie not identified. & A & & $x$ & & $x$ & & & & $x$ & & $x$ \\
\hline ORDER COLEOPTERA & & & & & & & & & & & \\
\hline Family Staphylinidae & & & & & & & & & & & \\
\hline Acylophorus sp. & PP & & $x$ & & $x$ & & $x$ & & $x$ & & $x$ \\
\hline Philonthus sp1. & PP & & $x$ & & $x$ & & $x$ & & $x$ & & $x$ \\
\hline Philonthus sp2. & PP & & $x$ & & $x$ & & $x$ & & $x$ & & $x$ \\
\hline Philonthus sp3. & PP & & & & $x$ & & $x$ & & $x$ & & $x$ \\
\hline Family Silphidae & & & & & & & & & & & \\
\hline Oxelytrum cayennense & $\mathrm{N}, \mathrm{PP}$ & & & & $x$ & & $x$ & & $x$ & & \\
\hline Family Histeridae & & & & & & & & & & & \\
\hline Hister sp. & PP & & $x$ & & $x$ & & $x$ & & $x$ & & $x$ \\
\hline Family Scarabaeidae & & & & & & & & & & & \\
\hline Onthophagus sp1. & $\mathrm{N}$ & & & & $x$ & & $x$ & & $x$ & & $x$ \\
\hline Onthophagus sp2. & N & & & & $x$ & & $x$ & & $x$ & & $x$ \\
\hline Onthophagus sp3. & N & & & & $x$ & & $x$ & & $x$ & & $x$ \\
\hline Onthophagus sp4. & $\mathrm{N}$ & & & & $x$ & & & & $x$ & & $x$ \\
\hline
\end{tabular}


Table 1. Continued

\begin{tabular}{|c|c|c|c|c|c|c|c|c|c|c|c|}
\hline \multirow{3}{*}{ Species } & \multirow{3}{*}{ CAT } & \multicolumn{10}{|c|}{ Stages of descomposition - Hours } \\
\hline & & \multirow{2}{*}{$\begin{array}{l}\text { Fresh } \\
\end{array}$} & \multirow{2}{*}{$\frac{0-15}{\mathrm{~A}}$} & \multicolumn{2}{|c|}{ Bloated 20-80 } & \multicolumn{2}{|c|}{ Active 85-115 } & \multicolumn{2}{|c|}{ Advanced 120-170 } & \multicolumn{2}{|c|}{ Remains $175-545$} \\
\hline & & & & I & A & I & A & 1 & A & 1 & A \\
\hline Onthophagus sp5. & $\mathrm{N}$ & & & & & & $x$ & & & & $x$ \\
\hline Ontherus sp. & $\mathrm{N}$ & & & & $x$ & & & & & & $x$ \\
\hline Eurysternus sp. & $\mathrm{N}$ & & & & $x$ & & $x$ & & $x$ & & $x$ \\
\hline Coprophanaeus sp. & $\mathrm{N}$ & & & & & & & & & & $x$ \\
\hline Deltochilum icarus & $\mathrm{N}$ & & & & $x$ & & & & & & $x$ \\
\hline Dichotomius sp. & $\mathrm{N}$ & & & & & & & & & & $x$ \\
\hline \multicolumn{12}{|l|}{ Family Trogidae } \\
\hline Unidentified species. & $\mathrm{N}$ & & & & & & & & $x$ & & $x$ \\
\hline Polipochila sp. & PP & & $x$ & & & & & & $x$ & & $x$ \\
\hline Unidentified species. & PP & & & & & & $x$ & & & & $x$ \\
\hline \multicolumn{12}{|l|}{ Family Chrysomelidae } \\
\hline Chrysolina fastuosa & A & & $x$ & & & & & & & & $x$ \\
\hline \multicolumn{12}{|l|}{ Family Coccinellidae } \\
\hline Hyperaspis erythrocephala & A & & & & & & & & & & $x$ \\
\hline \multicolumn{12}{|l|}{ Family Curculionidae } \\
\hline Nicentrus decipiens & A & & $x$ & & $x$ & & & & & & $x$ \\
\hline Phloeborus punctatorugosus & A & & & & $x$ & & & & & & $x$ \\
\hline \multicolumn{12}{|l|}{ Family Passalidae } \\
\hline Passalus interruptus & A & & & & & & $x$ & & & & $x$ \\
\hline \multicolumn{12}{|l|}{ Family Melolonthidae } \\
\hline Apogonia sp. & A & & & & $x$ & & $x$ & & & & $x$ \\
\hline Family Elmidae & & & & & & & & & & & \\
\hline Unidentified species. & A & & & & & & & & & & \\
\hline ORDER HYMENOPTERA & & & & & & & & & & & \\
\hline Family Formicidae & & & & & & & & & & & \\
\hline Camponotus sp. & 0 & & $x$ & & $x$ & & $x$ & & $x$ & & $x$ \\
\hline Cephalotes sp. & 0 & & $x$ & & $x$ & & $x$ & & $x$ & & $x$ \\
\hline Cheliomyrmex sp. & 0 & & $x$ & & $x$ & & $x$ & & $x$ & & $x$ \\
\hline Dinoponera sp. & 0 & & $x$ & & $x$ & & $x$ & & $x$ & & $x$ \\
\hline Labidus sp. & 0 & & & & $x$ & & $x$ & & & & $x$ \\
\hline Lasiophanes sp1. & 0 & & $x$ & & $x$ & & $x$ & & $x$ & & $x$ \\
\hline Lasiophanes sp2. & 0 & & $x$ & & $x$ & & & & $x$ & & $x$ \\
\hline Solenopsis sp. & 0 & & $x$ & & $x$ & & $x$ & & $x$ & & $x$ \\
\hline Myrcidris sp. & 0 & & $x$ & & $x$ & & & & $x$ & & $x$ \\
\hline Neivamyrmex sp. & 0 & & $x$ & & $x$ & & & & & & $x$ \\
\hline Prionopelta sp. & 0 & & & & & & $x$ & & & & \\
\hline Anoplolepis sp. & 0 & & & & & & & & & & $x$ \\
\hline Phaneroserphus sp. & 0 & & & & $x$ & & & & & & $x$ \\
\hline Family Vespidae & & & & & & & & & & & \\
\hline Hypalastoroides sp1. & PP & & $x$ & & $x$ & & & & & & \\
\hline Polybiasp. & PP & & & & $x$ & & $x$ & & $x$ & & $x$ \\
\hline Trimeria sp. & PP & & $x$ & & $x$ & & $x$ & & $x$ & & $x$ \\
\hline Family Tiphidae & & & & & & & & & & & \\
\hline Aelurus sp. & PP & & & & $x$ & & & & & & \\
\hline Methocha sp. & PP & & & & $x$ & & & & & & \\
\hline Myzinun sp. & PP & & & & & & $x$ & & & & \\
\hline Paratiphia sp. & PP & & & & & & & & $x$ & & \\
\hline Polybia sp. & PP & & & & & & & & & & $x$ \\
\hline Tiphia sp. & PP & & & & $x$ & & $x$ & & $x$ & & $x$ \\
\hline Family Diapriidae & & & & & & & & & & & \\
\hline Phaneroserphus sp. & PP & & & & $x$ & & & & & & $x$ \\
\hline Unidentified species. & PP & & & & & & & & & & $x$ \\
\hline Family Chalcididae & & & & & & & & & & & \\
\hline Belaspidia sp. & PP & & & & $x$ & & & & & & \\
\hline
\end{tabular}


Table 1. Continued

\begin{tabular}{|c|c|c|c|c|c|c|c|c|c|c|c|}
\hline \multirow{3}{*}{ Species } & \multirow{3}{*}{ CAT } & \multicolumn{10}{|c|}{ Stages of descomposition - Hours } \\
\hline & & \multirow{2}{*}{$\begin{array}{l}\text { Fresh } \\
\end{array}$} & \multirow{2}{*}{$\frac{0-15}{A}$} & \multicolumn{2}{|c|}{ Bloated 20-80 } & \multicolumn{2}{|c|}{ Active 85-115 } & \multicolumn{2}{|c|}{ Advanced 120-170 } & \multicolumn{2}{|c|}{ Remains $175-545$} \\
\hline & & & & I & A & I & A & 1 & A & 1 & A \\
\hline \multicolumn{12}{|l|}{ Family Evanniidae } \\
\hline Evaniella sp. & PP & & & & $x$ & & & & & & \\
\hline Semaeomyia sp. & PP & & & & & & & & & & $x$ \\
\hline \multicolumn{12}{|l|}{ Family Braconidae } \\
\hline Unidentified species. & PP & & & & & & $x$ & & $x$ & & $x$ \\
\hline \multicolumn{12}{|l|}{ Family Halictidae } \\
\hline Augochlorini sp. & A & & & & & & & & & & $x$ \\
\hline \multicolumn{12}{|c|}{ Family Ichneumonidae } \\
\hline Unidentified species. & PP & & & & $x$ & & & & & & \\
\hline \multicolumn{12}{|l|}{ Family Megachilidae } \\
\hline Unidentified species. & PP & & & & $x$ & & & & & & $x$ \\
\hline \multicolumn{12}{|l|}{ ORDER ARANEAE } \\
\hline Family Ctenidae & & & & & & & & & & & \\
\hline Unidentified species. & PP & & $x$ & & $x$ & & $x$ & & $x$ & & $x$ \\
\hline Family Pisauridae & & & & & & & & & & & \\
\hline Unidentified species. & PP & & & & $x$ & & & & $x$ & & $x$ \\
\hline Family Clubionidae & & & & & & & & & & & \\
\hline Unidentified species. & PP & & $x$ & & $x$ & & & & & & $x$ \\
\hline Family Salticidae & & & & & & & & & & & \\
\hline Unidentified species. & PP & & & & $x$ & & & & & & $x$ \\
\hline Family Oxyopidae & & & & & & & & & & & \\
\hline Unidentified species. & PP & & $x$ & & & & & & & & $x$ \\
\hline ORDER BLATTODEA & & & & & & & & & & & \\
\hline Family Blattidae & & & & & & & & & & & \\
\hline Unidentified species. & $\mathrm{N}$ & & $x$ & $x$ & $x$ & $x$ & $x$ & $x$ & $x$ & $x$ & $x$ \\
\hline ORDER HEMIPTERA & & & & & & & & & & & \\
\hline Family Cicadellidae & & & & & & & & & & & \\
\hline Unidentified species. & A & & $x$ & & $x$ & & $x$ & & $x$ & & $x$ \\
\hline Family Coreidae & & & & & & & & & & & \\
\hline Unidentified species. & A & & $x$ & & & & & & $x$ & & $x$ \\
\hline Family Cydnidae & & & & & & & & & & & \\
\hline Geoutomus pygmaeus & A & & $x$ & & $x$ & & & & $x$ & & $x$ \\
\hline Family Membracida & & & & & & & & & & & \\
\hline Cyphonia sp. & A & & & & & & & & & & \\
\hline Heteronotus sp. & A & & & & $x$ & & & & & & \\
\hline Family Pentatomida & & & & & & & & & & & \\
\hline Unidentified species. & A & & & & & & & & $x$ & & \\
\hline Family Tropiduchida & & & & & & & & & & & \\
\hline Unidentified species. & A & & $x$ & & $x$ & & $x$ & & $x$ & & $x$ \\
\hline ORDER ORTHOPTER & & & & & & & & & & & \\
\hline Family Eumastacidae & & & & & & & & & & & \\
\hline Unidentified species. & A & & & & $x$ & & $x$ & & $x$ & & $x$ \\
\hline Family Gryllacridida & & & & & & & & & & & \\
\hline Unidentified species. & A & & $x$ & & $x$ & & $x$ & & $x$ & & $x$ \\
\hline Family Gryllidae & & & & & & & & & & & \\
\hline Unidentified species. & A & & & & $x$ & & & & $x$ & & $x$ \\
\hline Family Gryllotalpida & & & & & & & & & & & \\
\hline Unidentified species. & A & & & & & & & & & & $x$ \\
\hline ORDER DERMAPTER & & & & & & & & & & & \\
\hline Family Labiidae & & & & & & & & & & & \\
\hline Unidentified species. & PP & & & $x$ & & & & & & & \\
\hline ORDER LEPIDOPTER & & & & & & & & & & & \\
\hline Unidentified family. & A & & $x$ & & $x$ & & $x$ & & $x$ & & $x$ \\
\hline
\end{tabular}


Table 2. Species composition and abundance (considering adult individuals) of Diptera, Coleoptera and Hymenoptera of greatest forensic importance in the cadaveric decomposition of pigs in a rural area of the Andean Amazon, Caquetá, Colombia. Numbers below the decomposition stage indicate the duration of the stage in hours from 0 to 545 . Values are the number of individuals, followed by the frequency $(\%$, in parentheses) in relation to the total number of individuals recorded throughout the study

\begin{tabular}{lccccc}
\hline \multirow{2}{*}{ Species } & Fresh & Bloated & Active & Advanced & Remains \\
\cline { 2 - 6 } & $(0-15)$ & $(20-80)$ & $(85-115)$ & $(120-170)$ & $(175-545)$ \\
\hline ORDER DIPTERA & & & &
\end{tabular}

\section{Family Calliphoridae}

Cocliomyia macellaria

(20)

Chrysomya albiceps

$0 \quad 20(0.06)$

$34(0.11)$

$48(0.15)$

$9(0.02)$

Paralucilia paraensis

0

$15(0.04)$

43 (0.13)

$27(0.08)$

49 (0.15)

Hemilucilia semidiaphana

$70(0.22)$

$77(0.24)$

$77(0.24)$

$20(0.06)$

Lucilia eximia

0

$9(0.02)$

$1(0.003)$

$4(0.01)$

1 (0.003)

Choroprocta idioidea

$7(0,02)$

$6(0.01)$

0

0

$6(0.01)$

\section{Family Sarcophagidae}

Tricharaea sp.
Oxysarcodexia sp.
Blaesoxipha sp.
Microcerella sp.
Peckia sp.
Sarcodexia sp1.
Sarcodexia sp2.
Sarcophaga sp.
Boettcheria sp.

$0 \quad 4(0.01)$

1 (0.003)

0

0

$2(0,006)$

$29(0.09)$

$12(0.03)$

$6(0.01)$

$12(0.03)$

$14(0.04)$

$25(0.08)$

0

$1(0.003)$

$11(0.03)$

$12(0.03)$

14 (0.04)

$\begin{array}{ll}0 & 1(0.003)\end{array}$

1 (0.003)

0

$10(0.03)$

$0 \quad 1(0.003)$

2 (0.006)

$0 \quad 3(0.009)$

0

0

0

$0 \quad 0$

$0 \quad 1(0.003)$

$1(0.003)$

$2(0.006)$

$10(0.03)$

0

$\begin{array}{ll}0 & 1(0.003 \\ 0 & 0\end{array}$

$\begin{array}{cc}1(0.003) & 10(0.03) \\ 1(0.003) & 0\end{array}$

$\begin{array}{ll}0 & 2(0.006)\end{array}$

0

0

0

\section{Family Muscidae}

Ophyra aenescens
Biopyrellia bipuncta
Trichomorellia flavipalpis

$6(0,01)$

$\begin{array}{llll}19(0.06) & 13(0.04) & 238(0.77) & 84(0.27) \\ 6(0.01) & 15(0.04) & 16(0.05) & 22(0.07) \\ 7(0.02) & 5(0.01) & 17(0.05) & 8(0.02)\end{array}$

\section{Family Piophilidae}

\begin{tabular}{|c|c|c|c|c|c|}
\hline Piophila sp. & 0 & $3(0.009)$ & 0 & $5(0.01)$ & $13(0.04)$ \\
\hline \multicolumn{6}{|l|}{ ORDER COLEOPTERA } \\
\hline \multicolumn{6}{|l|}{ Family Scarabaeidae } \\
\hline Onthophagus sp1. & 0 & $18(0.05)$ & $4(0.012)$ & $11(0.03)$ & $62(0.20)$ \\
\hline Onthophagus sp2. & 0 & $13(0.04)$ & $9(0.02)$ & $12(0.03)$ & $131(0.42)$ \\
\hline Onthophagus sp3. & 0 & $12(0.03)$ & $7(0.02)$ & $10(0.03)$ & $69(0.22)$ \\
\hline Onthophagus sp4. & 0 & $1(0.003)$ & 0 & $2(0.006)$ & $25(0.08)$ \\
\hline Onthophagus sp5. & 0 & 0 & $1(0.003)$ & 0 & $5(0.01)$ \\
\hline Eurysternus sp. & 0 & $5(0.01)$ & $4(0.01)$ & $1(0.003)$ & $2(0.006)$ \\
\hline Ontherus sp. & 0 & $1(0.003)$ & 0 & 0 & $5(0.01)$ \\
\hline Deltochilum icarus & 0 & $1(0.003)$ & 0 & 0 & $15(0.04)$ \\
\hline Dichotomius sp. & 0 & 0 & 0 & 0 & $6(0.01)$ \\
\hline Coprophanaeus sp. & 0 & 0 & 0 & 0 & $7(0.02)$ \\
\hline \multicolumn{6}{|l|}{ Family Silphidae } \\
\hline Oxelytrum cayennense & 0 & $1(0.003)$ & $2(0.006)$ & $4(0.01)$ & 0 \\
\hline
\end{tabular}


Table 2. Continued

\begin{tabular}{|c|c|c|c|c|c|}
\hline \multirow{2}{*}{ Species } & Fresh & Bloated & Active & Advanced & Remains \\
\hline & $(0-15)$ & $(20-80)$ & $(85-115)$ & $(120-170)$ & $(175-545)$ \\
\hline \multicolumn{6}{|l|}{ Family Histeridae } \\
\hline Hister sp. & $1(0.003)$ & $34(0.11)$ & $63(0.20)$ & $196(0.63)$ & $1680(5.44)$ \\
\hline \multicolumn{6}{|c|}{ Family Staphylinidae } \\
\hline Acylophorus sp. & $1(0.003)$ & $3(0.009)$ & $1(0.003)$ & $7(0.02)$ & $20(0.06)$ \\
\hline Philonthus sp1. & $1(0.003)$ & $153(0.49)$ & $96(0.31)$ & $225(0.72)$ & $506(1.64)$ \\
\hline Philonthus sp2. & $1(0.003)$ & $27(0.08)$ & $4(0.01)$ & $10(0.03)$ & $111(0.36)$ \\
\hline Philonthus sp3. & 0 & $13(0.04)$ & $5(0.01)$ & $25(0.08)$ & $20(0.06)$ \\
\hline \multicolumn{6}{|c|}{ ORDER HYMENOPTERA } \\
\hline \multicolumn{6}{|l|}{ Family Formicidae } \\
\hline Camponotus sp. & $967(3.13)$ & $588(1.90)$ & $63(0.20)$ & $101(0.32)$ & $620(2.01)$ \\
\hline Cephalotes sp. & $1(0.003)$ & $9(0.02)$ & $1(0.003)$ & $2(0.006)$ & $33(0.10)$ \\
\hline Cheliomyrmex sp. & $11(0.35)$ & $15(0.48)$ & $8(0.02)$ & $15(0.14)$ & $64(0.20)$ \\
\hline Dinoponera sp. & $15(0.04)$ & $18(0.05)$ & $8(0.02)$ & $5(0.01)$ & $69(0.22)$ \\
\hline Labidus sp. & 0 & $16(0.05)$ & $1(0.003)$ & 0 & $83(0.26)$ \\
\hline Lasiophanes sp1. & $2(0.006)$ & $10(0.03)$ & $5(0.01)$ & $1(0.003)$ & $2(0.006)$ \\
\hline Lasiophanes sp2. & $3(0.009)$ & $4(0.01)$ & 0 & $5(0.01)$ & $11(0.03)$ \\
\hline Myrcidris sp. & $3(0.009)$ & $2(0.006)$ & 0 & $2(0.006)$ & $5(0.01)$ \\
\hline Neivamyrmex sp. & $32(0.10)$ & $36(0.11)$ & 0 & 0 & $1(0.003)$ \\
\hline Solenopsis & $31(0.10)$ & $131(0.42)$ & $1(0.003)$ & $4(0.01)$ & $3(0.009)$ \\
\hline Anoplolepis sp. & 0 & 0 & 0 & 0 & $1(0.003)$ \\
\hline Phaneroserphus sp. & 0 & $6(0.01)$ & 0 & 0 & $5(0.01)$ \\
\hline
\end{tabular}

Table 3. Immature individuals of Diptera species associated to the cadaveric decomposition of pigs in a rural area of the Andean Amazon, Caquetá, Colombia. Numbers below the decomposition stage indicate the duration of the stage in hours from 0 to 545 . Values are the number of individuals, followed by the frequency (\% in parentheses) in relation to the total number of individuals recorded throughout the study.

\begin{tabular}{|c|c|c|c|c|c|}
\hline \multirow{2}{*}{ Species } & Fresh & Bloated & Active & Advanced & Remains \\
\hline & $(0-15)$ & $(20-80)$ & $(85-115)$ & $(120-170)$ & (175-545) \\
\hline
\end{tabular}

\section{ORDER DIPTERA}

\section{Family Calliphoridae}

\begin{tabular}{lccccc} 
Chrysomya albiceps & 0 & 0 & 0 & $1920(6.22)$ & 2858 (9.26) \\
Cocliomyia macellaria & 0 & $36(0.11)$ & 0 & $2700(8.75)$ & $444(1.44)$ \\
\hline Family Muscidae & & & & & \\
Hemilucilia semidiaphana & 0 & $21(0.06)$ & $22(0.07)$ & $67(0.21)$ & $757(2.45)$ \\
Ophyra aenescens & 0 & 0 & $2(0.006)$ & $11(0.03)$ & $9542(30.94)$
\end{tabular}



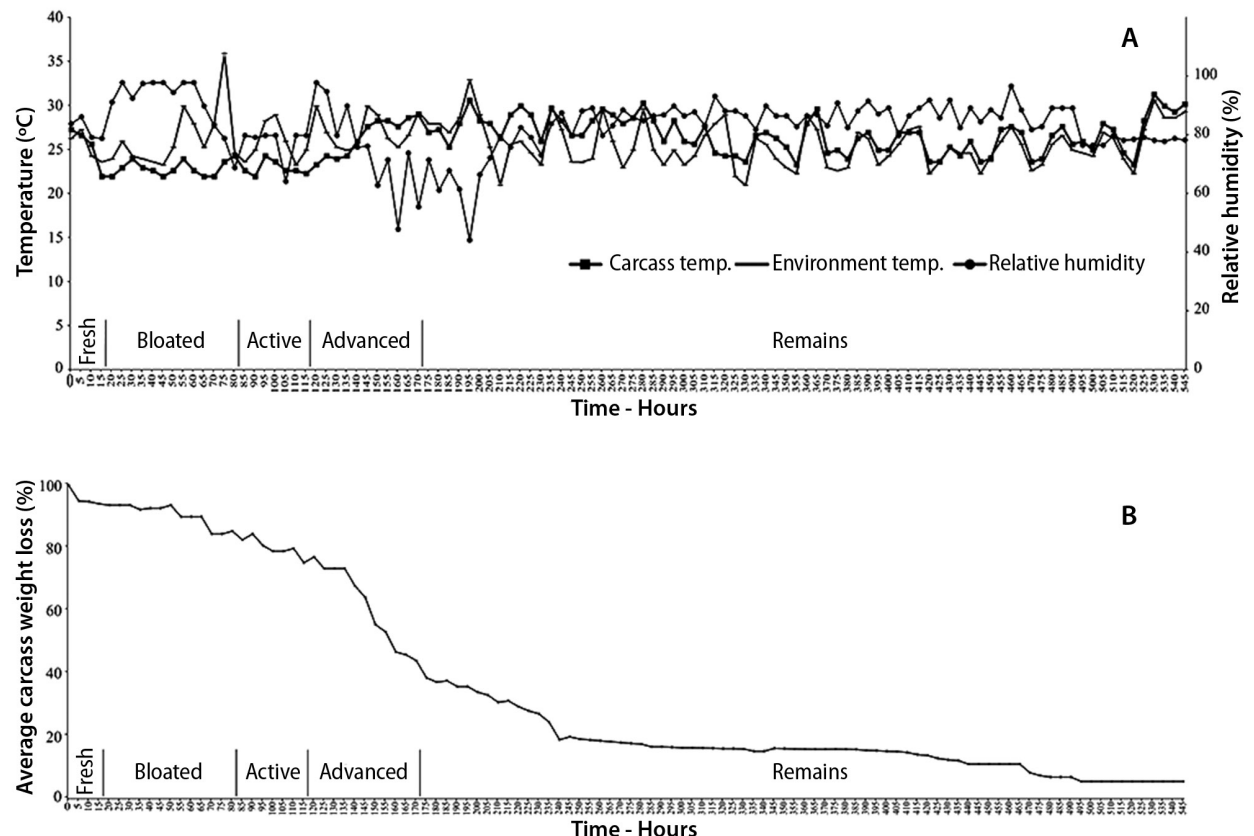

Figure 1. Daily variation of environmental variables (temperature and relative humidity) and carcass decay variables throughout the decomposition period of three pig carcasses in a rural area of the Andean Amazon, Caquetá, Colombia. A - daily variation of mean air temparature, mean relative humidity and mean body temperature of the carcasses; B - mean daily proportion of weight loss of the three carcasses. The stages of decomposition are indicated in relation to the total duration period in days.

other liquids typical of the decomposition to be observed, which still do not allow bones to be exposed. This stage was characterized by the presence of large quantities od immature dipterans, mainly C. macellaria and C. albiceps. Immatures of $H$. semidiaphana and $O$. aenescens, as well as nymphs of Blattidae, were also present (Tables 1 and 3, Supplementary Material, Table S1). Rectal carcass temperature varied between 23.33 and $29^{\circ} \mathrm{C}$, while environmental temperature ranged between 25.33 and $30^{\circ} \mathrm{C}$, relative humidity oscillated between $48 \%$ and $98 \%$ (Figure 1a), and carcass weight loss was of $31.19 \%$, for an accumulated total of $55.97 \%$ (Figure 1b).

Stage 5 - carcass remains (hour 175 to 545) - This phase started when the carcass was reduced to skin, hairs and bones. The original form of the carcass was not identificable because the remains were dispersed by the degradation process. It was also characterized by the absence of odors. The entomofauna was characterized by a predominance of immature of Diptera, mainly $O$. aenescens (LII, LIII, prepupa) (Muscidae), and $C$. albiceps (LII, LIII, prepupa, pupa, empty puparium), followed by H. semidiaphana (LIII), C. macellaria (LIII, prepupa) (Calliphoridae), and nymphs of Blattidae (Tables 1 and 3, Supplementary Material, Table S1). Among adult insects Coleoptera were the most abundant, followed by Formicidae and Diptera (Tables 1 and 2, Supplementary Material, Table S1). Rectal carcass temperature fluctuated between 23.33 and $30.67^{\circ} \mathrm{C}$, while environmental temperature ranged between 21 and $33{ }^{\circ} \mathrm{C}$, relative humidity varied between $44.33 \%$ and $97 \%$ (Figure 1a), and weight loss was of $38.53 \%$, for an accumulated total of $94.5 \%$ (Figure $1 \mathrm{~b}$ ).

\section{Occurrence matrix}

The occurrence matrix was developed based on the species that were directly related to the decomposition of the carcass. Coclhiomyia macellaria was the colonizing species in larval stage, appearing from hour 50 (day 2) until hour 265 (day 11), initiating its post-feeding migration in hour 160 (day 7), and appearing only sporadically after that until hour 525 (day 22). Paralucilia paraensis was present only in the adult stage, from hour 30 (day 2) until hour 425 (day 17). Species present both in the immature and adult stages were $H$. semidiaphana, from hour 35 (day 2) until hour 340 (day 15), C. albiceps, from hour 25 (day 2) until hour 545 (day 23), and O. aenescens, from hour 80 (day 4) until hour 535 (day 22). Ants influenced the time of decomposition of the carcasses. Camponotus sp. was the main species responsible for delaying colonization, and it was observed preying on eggs and larvae of Diptera from stage 1 (fresh) to stage 5 (remains) (Supplementary Material, Table S1).

\section{DISCUSSION}

Five decomposition stages were observed, which coincide with other studies in rural areas in the United States (Early and Goff 1986, Goff 1992) and in Colombia (Grisales et al. 2010, Ramos-Pastrana and Wolff 2011). The same number of stages was reported for urban environments (Wolff et al. 2001), the Paramo (Martínez et al. 2007) and in enclosed conditions (Ramos-Pastrana et al. 2014) in Colombia.

Total time from stage 1 (fresh) to stage 5 (remains) lasted 545 $\mathrm{h}$ (22.7 days), differing from studies in other regions of Colombia 
(36 days, Pérez et al. 2005; 97 days, Segura et al. 2009; 26 days, Grisales et al. 2010). These differences can be attributed to the geographic and climatic differences of the regions where these studies were developed. In comparison to other regions, the Amazon is marked by a higher diversity and abundance of insects.

Ants played a determining role in the duration of the decomposition period in our study area. The process was slowed down by the removal of large quantities of dipteran eggs by ant predation, mainly by Camponotus sp. The same phenomenon was observed by Wells and Greenberg (1994), Stoker et al. (1995), Anderson and Vanlaerhoven (1996), Perez et al. (2005), and may affect the normal succession pattern in the decomposition process, as well as the indicator species for the determination of PMI.

The general pattern of succession showed that, as the decay advanced, Diptera were followed by Coleoptera, which agrees with reports from other studies in rural (Centeno $\mathrm{et} \mathrm{al}$. 2002; Grisales et al. 2010; Ramos-Pastrana and Wolff 2011), semi-rural (Segura et al. 2009, 2011), paramo (Martínez et al. 2007), and urban areas (Wolff et al. 2001, Pérez et al. 2005) in Colombia, and in other countries in the Neotropics (Vasconcelos and Araujo 2012) and the Nearctic (Anderson and VanLaerhoven 1996). With nine orders, 46 families, 95 genera and 106 species, our study reported a much higher diversity of taxa than that reported by another study in the Andean Amazon, Caquetá, Colombian, where only five orders, 20 families, 33 genera and 33 species were reported (Ramos-Pastrana and Wolff 2011), which can be attributed to methodological differences between the studies. In this study the number of biomodels and daily samplings was increased.

Cochliomya macellaria, having appeared in larval stage from hour 50 (day 2), is a useful indicator species for the determination of PMI in the study area, coinciding with what had been reported by Ramos-Pastrana and Wolff (2011), and confirming that this species behaves as a colonizing species of corpses in rural areas of the Colombian Amazonian Piedmont. This contrasts with other areas of Colombia, where the colonizing species reported were Lucilia eximia, in the rural area of Pereira (Grisales et al. 2010), L. sericata (Meigen 1826), in the urban area of Medellín (Pérez et al. 2005), Calliphora nigribasis (Macquart 1851) and Compsomyiops verena (Walter 1849), in the Chingaza National Park (Martínez et al. 2007).

\section{CONCLUSIONS}

This study provides new information to improve the methods for accurately estimating post-mortem interval (PMI) in the Amazonian Piedmont of Colombia, and contributes to the knowledge on forensic science in Colombia. Once the colonization on the pig carcasses was consolidated, C. macellaria, $C$. albiceps, $H$. semidiaphana and $O$. aenescens immatures were considered as the main organisms responsible for consumption of decaying tissue and for accelerating the process of decomposition. Furthermore, the study area influenced the decomposition, with differences observed in the times of each decomposition stage and in the associated fauna when compared to other studies of this kind. This study reflects the importance of determining and interpreting succession patterns of species of forensic importance in each geographic region. In Colombia, given that ecological conditions are so variable within the country, it is not possible to extrapolate results from one region to another. This type of study is very important for the advancement of forensic science in Colombia, with special relevance as a tool for determination PMI in cases of human death.

\section{ACKNOWLEDGMENTS}

To Universidad de la Amazonia and Colciencias Project 6012009, to Semillero de Investigación en Entomología (SIEN) of the Universidad de la Amazonia, to Centro de Investigaciones INBIANAM, the Universidad de Antioquia, and biologists Edna Lucia Ospina for their contributions in the field, to biologists Julian Penagos for the edition of the figure.

\section{REFERENCES}

Anderson, G.S. 2010. Factors That Influence Insect Succession on Carrion. In: Byrd, J.H.; Castner, J.L. (Ed.). Forensic Entomology: The Utility of Arthropods in Legal Investigations. 2nd ed. CRC Boca Raton, p.201-250.

Anderson, G.S.; Vanlaerhoven, S.L. 1996. Initial studies on insect succession on Carrion in Southwestern British Columbia. Journal of Forensic Science, 41: 617-625.

Archer, M.S. 2003. Annual variation in arrival and departure times of carrion insects at carcasses: implications for sucession studies in forensic entomology. Australian Journal of Zoology, 51: 569-576.

Brwon, BV, Borkent A, Cumming JM, Wood DM, Zumbado MA 2009. Manual of Central American Diptera. vol. 1, NCR, Research Press, Ottawa, 714p.

Brwon BV, Borkent A, Cumming JM, Wood DM, Woodley NE, Zumbado MA 2010. Manual of Central American Diptera. vol 1, NCR, Research Press, 728p.

Carvalho, L.M.L.; Thyssen, P.J.; Goff, M.L.; Linhares, L.A.X. 2004. Observation on the succession patterns of necrophagous insects on a pig carcass in an urban area of southeastern Brazil. Journal of Forensic Medical Toxicology, 5: 33-39.

Carvalho, C.J.B.; Rafael, J.A.; Couri, S.M. 2012. Diptera. In: Rafael, J.A.; Melo, G.A.R.; Carvalho, C.J.B.; Casari, S.A.; Constantino, R. (Ed.). Insetos do Brasil, Diversidade e Taxonomia, Riberao Preto, Holos Editora, p.701-743.

Catts, E.P.; Haskell, N.H. 1990. Entomology and death: A procedural guide. Joy's Print Shop, Inc, Clemson, South Caroloina, 182p.

Centeno, N.D.; Maldonado, M.; Oliva, A. 2002. Seasonal patterns occuring on sheltered and unsheltered pig carcasses in Buenos Aires province (Argentina). Forensic Science International, 126: 63-70.

CIMAZ - Centro de Investigaciones Amazonicas MACAGUAL. 2007. Poryecto de la Vicerrectoria de Investigaciones y Posgrados de la Universidad de la Amazonia. 35p.

Cruz, T.M.; Vasconcelos, S.D. 2006. Entomofauna de solo associada à decomposição de carcaça de suíno em um fragmento de mata atlántica de Pernambuco, Brasil. Biociências, 14: 193-201. 
Dekeirsschieter, J.; Frederick, C.; Verheggen, F.J.; Drugman, D.; Hambruge, E. 2013. Diversity of Forensic rove beetles (Coleoptera: Staphylinidae) associated with decay pig carcass in a forest biotope. Journal of Forensic Science, 4: 1032-1040.

Early, M.; Goff, M.L. 1986. Arthropod succession patterns in exposed carrion on the Island of Oáhu, Hawaii island, USA. Journal of Medical Entomology, 23: 520-531.

Fernández, F. 2003. Introducción a las hormigas de la región neotropical. Instituto de Investigación de Recursos Biológicos Alexander Von Humboldt, Bogotá, Colombia, 398p.

Fernández, F.; Sharkey, M.J. 2006. Introducción a los Hymenoptera de la región neotropical. Sociedad Colombiana de Entomología y Universidad Nacional de Colombia, Bogotá D.C, 894p.

Flórez, E.; Wolff, M. 2009. Descripción y clave de los estadios inmaduros de las principales especies de Calliphoridae (Diptera) de importancia forense en Colombia. Neotropical Entomology, 38: 418-429.

Goff, M.L. 1992. Estimation of postmortem interval using arthropod successional patterns. Forensic Science Review, 5: 81-94.

Greenberg, B.; Szyska, M.L. 1984. Immature stages and biology of fifteen species of Peruvian calliphoridae (Diptera). Annals of the Entmological Society of America, 77: 488-517.

Grisales, D.; Ruiz, M.; Villegas, S. 2010. Insects associated with exposed decomposing bodies in the Colombian Andean Coffee Region. Revista Brasileira de Entomologia, 54: 637-644.

Haskell, N.H. 1990. Entomological Collection Techniques at Autopsy and for Specific Enviroments. In: Catts, E.P.; Haskell, N.H. (Ed.). Entomology \& Death: A Procedural Guide. Joyce's Print Shop, Inc, Clemson, South Carolina, p.98-110.

Hobischak, N.R.; Vanlaerhoven, S.L.; Anderson, G.S. 2006. Successional patterns of diversity in insect fauna on carrion in sun and shade in the Boreal Forest Region of Canada, near Edmonton, Alberta. Canadian Entomology, 138: 376-683.

Holdridge, L. 1996. Ecología basada en zonas de vida. Instituto Interamericano de Cooperación para la Agricultura, San José, Costa Rica, 78p.

IGAC - Instituto Geografico Agustin Codazzi. 2010. Caquetá características geográficas. Instituto Geográfico Agustín Codazzi, Bogotá, Colombia, 376p.

Magaña, C. 2001. La entomología forense y su aplicación a la medicina legal, data de la muerte. Boletin de la Sociedad Entomológica Aragonesa, 28: 49-57.

Martínez, E.; Duque, P.; Wolff, M. 2007. Succession pattern of carrion-feeding insects in Paramo, Colombia. Forensic Science International, 166: 182-189.

Navarrete-Heredia, J.L.; Newton, A.F.; Thayer, M.K.; Ashe, J.S.; Chandler, D.S. 2002. Guía ilustrada para los géneros de Staphylinidae (Coleoptera) de Mexico. Universidad de Guadalajara y CONABIO, México, 401p.

Payne, J.A. 1965. A summer carrion study of the baby pig Sus scrofa Linnaeus. Ecology, 46: 592-602.
Pérez, S.P.; Duque, P.; Wolff, M. 2005. Successional behaivor and occurrence matriz of carrion-asociate arthropods in the urban area of Medellin, Colombia. Journal of Forensic Science, 50: 448-454.

Pujol-Luz, J.R.; Marques, H.; Ururahy-Rodrigues, A.; Rafael, J.A.; Santana, F.H.A.; Arantes, L.C.; Constantino, R. 2006. A forensic entomology case from the Amazon rain forest of Brazil. Journal of Forensic Science, 51: 1151-1153.

Ramos-Pastrana, Y.; Wolff, M. 2011. Entomofauna Cadavérica Asociada a Cerdos Expuestos al Sol y Sombra, en el Piedemonte Amazónico Colombiano. Revista Momentos de Ciencia, 8: 45-54.

Ramos-Pastrana, Y.; Velasquez-Valencia, A.; Wolff, M. Preliminary study of insects associated to indoor body decay in Colombia. Revista Brasileira de Entomologia, 58: 326-332.

Salazar-Ortega, J. 2008. Study of successional entomofauna associated to domestic pig (Sus scrofa) corpse decomposition in field conditions. Universitas Scientiarum, 13: 21-32.

Segura, N.A.; Usaquén, W.; Sánchez, M.C.; Chuaire, L.; Bello, F. 2009. Succession pattern of cadaverous entomofauna in a semi-rural area of Bogota, Colombia. Forensic Science International, 187: 66-72.

Segura, N.A.; Bonilla, M.A.; Usaquen, W.; Bello, F. 2011. Entomofauna resourse distribution associated with pig cadaver in Bogotá DC. Medical and Veterinary Entomology, 25: 46-52.

Smith, K. 1986. A Manual of Forensic Entomology. Department of Entomology British Museum (Natural History), London, 203p.

Tullis, K.; Goff, M.L. 1987. Arthropod succession in exposed carrion in a tropical rainforest on O'ahu Island, Hawaii. Jornal of Medical Entomology, 24: 332-339.

Vasconcelos, S.D.; Araujo, M.C.S. 2012. Necrophagous species of Diptera and Coleoptera in northeastern Brazil: state of the art and challenges for the Forensic Entomologist. Revista Brasileira de Entomologia, 56: 7-14.

Wolff, M.; Uribe, A.; Ortiz, A.; Duque, P. 2001. A preliminary study of forensic entomology in Medellín, Colombia. Forensic Science International, 102: 53-59.

RECEIVED: $30 / 03 / 2017$

ACCEPTED: 08/12/2017

ASSOCIATE EDITOR: Claudia Keller

\section{SUPPLEMENTARY MATERIAL}

(only available in the electronic version)

RAMOS-PASTRANA et al. Insects of forensic importance associated to cadaveric decomposition in a rural area of the Andean Amazon, Caquetá, Colombia

Table S1. Matrix of occurrence of insects of forensic importance associated to cadaveric decomposition of pigs in a rural area of the Andean Amazon, Caquetá, Colombia. Cells highlighted in gray indicate the first appearance of the species. 0 : Absence, 1: Presence, LI: First instar larva LII: Second instar larva, LIII: Third instar larva, Pp: Prepupa, P: Pupa, A: Adult, Ppo: Puparium. 


\section{SUPPLEMENTARY MATERIAL (only available in the electronic version)}

RAMOS-PASTRANA et al. Insects of forensic importance associated to cadaveric decomposition in a rural area of the Andean Amazon, Caquetá, Colombia

Table S1. Matrix of occurrence of insects of forensic importance associated to cadaveric decomposition of pigs in a rural area of the Andean Amazon, Caquetá, Colombia. Cells highlighted in gray indicate the first appearance of the species. 0: Absence, 1: Presence, LI: First instar larva LII: Second instar larva, LIII: Third instar larva Pp: Prepupa, P: Pupa, A: Adult, Ppo: Puparium.

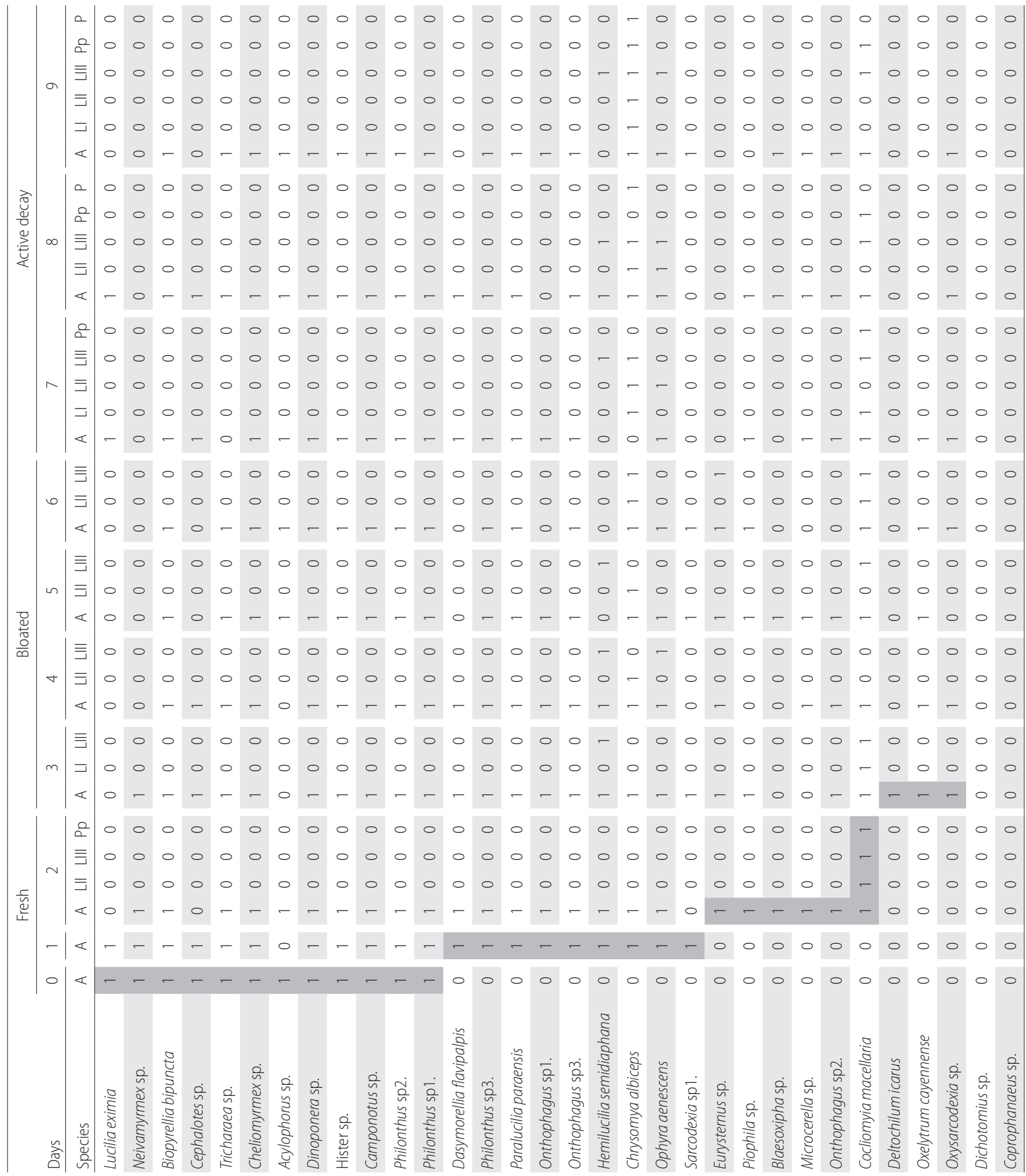


Table S1. Continued

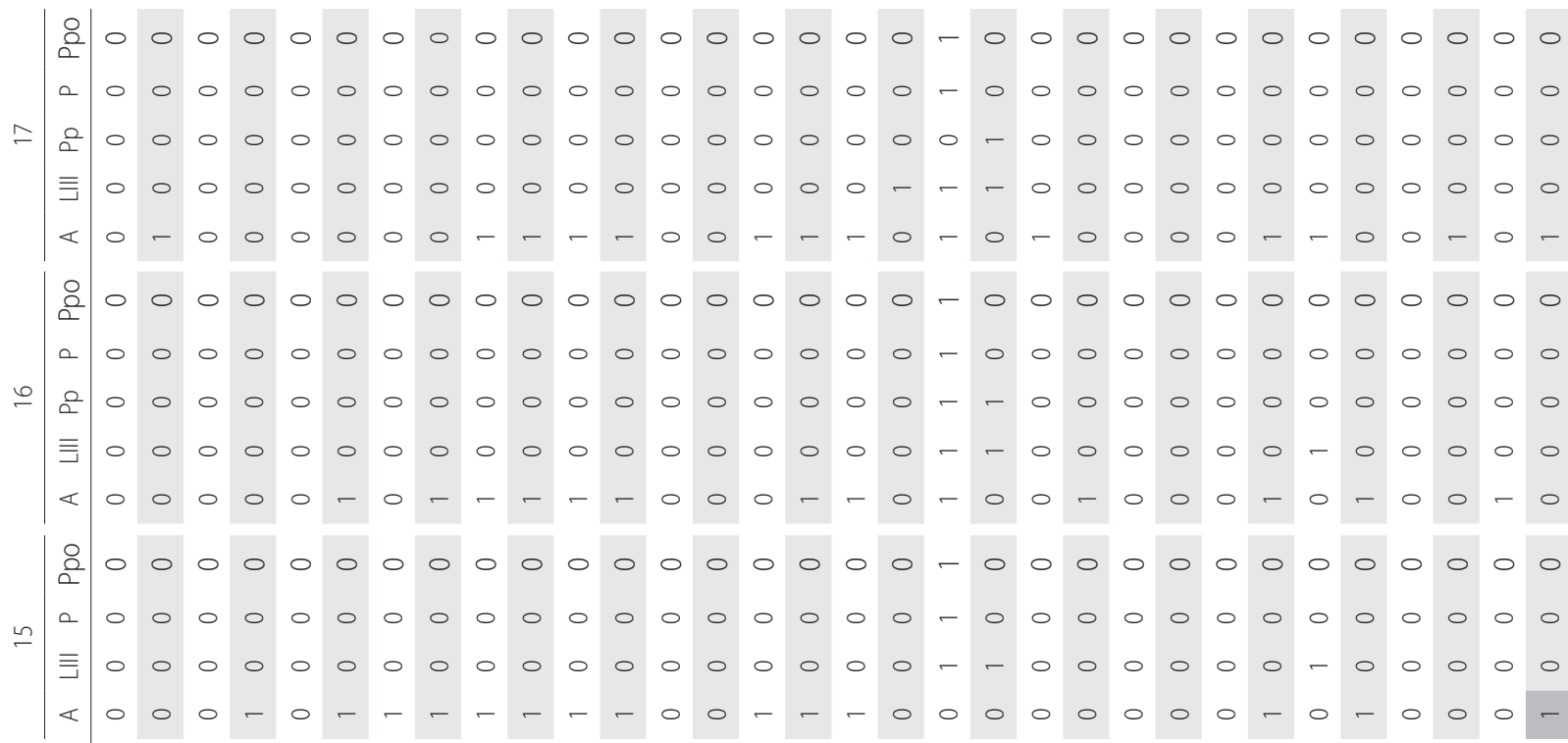

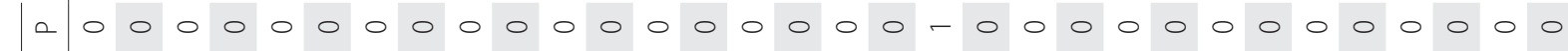

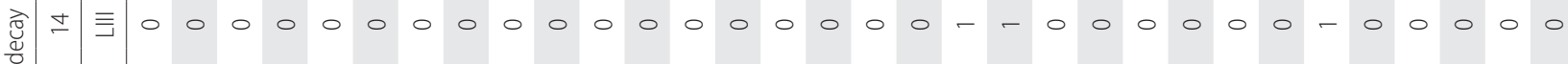

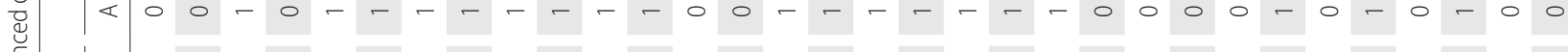

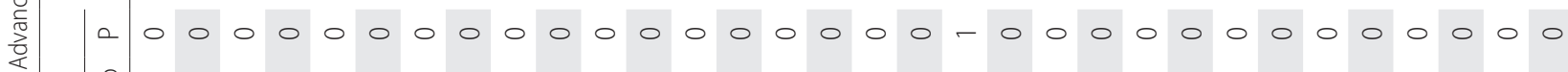

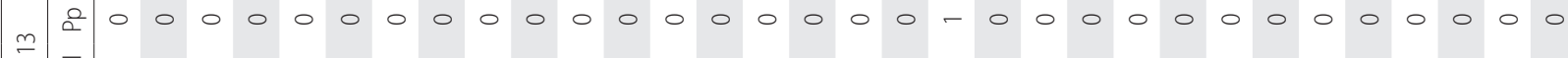

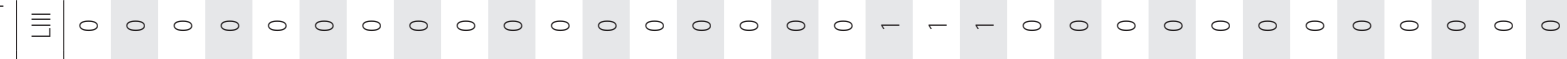
$<00--------0------0----000---000$

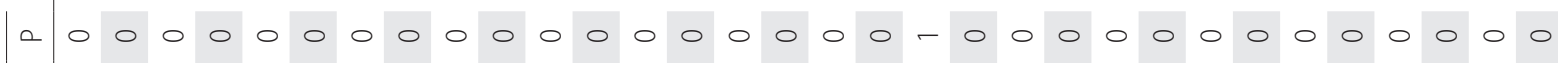

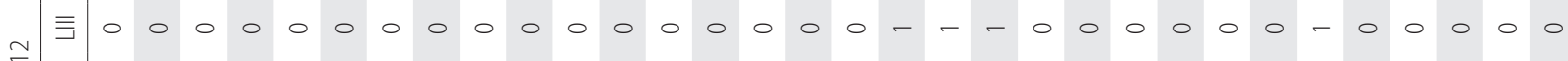

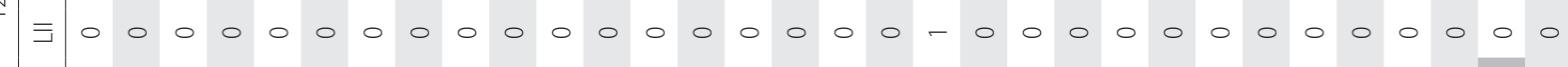
$<00----0-----0-0--0-0-00---000--0$

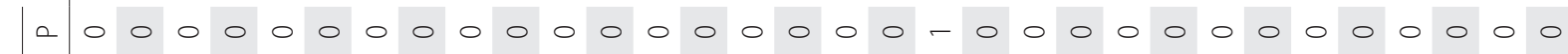

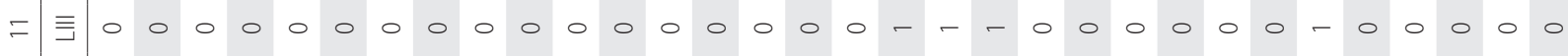
$<00----0-------00-000-0------0-0$

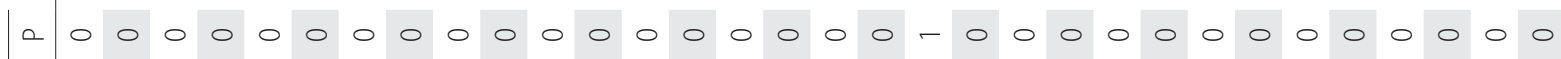
$\Omega$

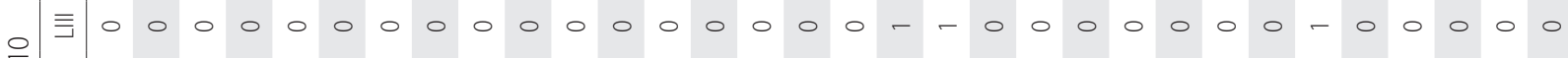

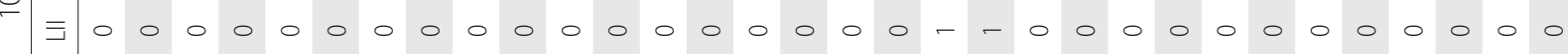

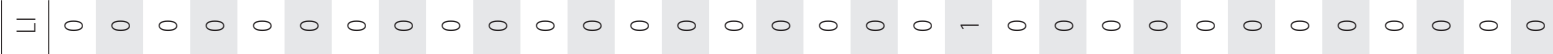

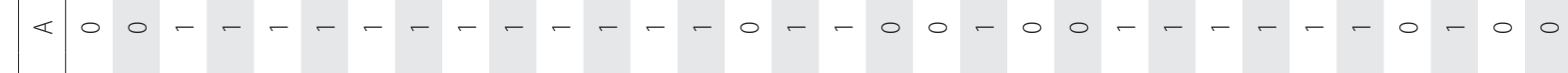

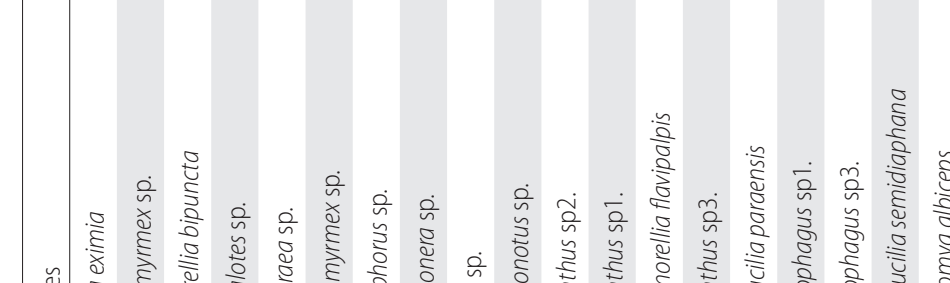


Table S1. Continued

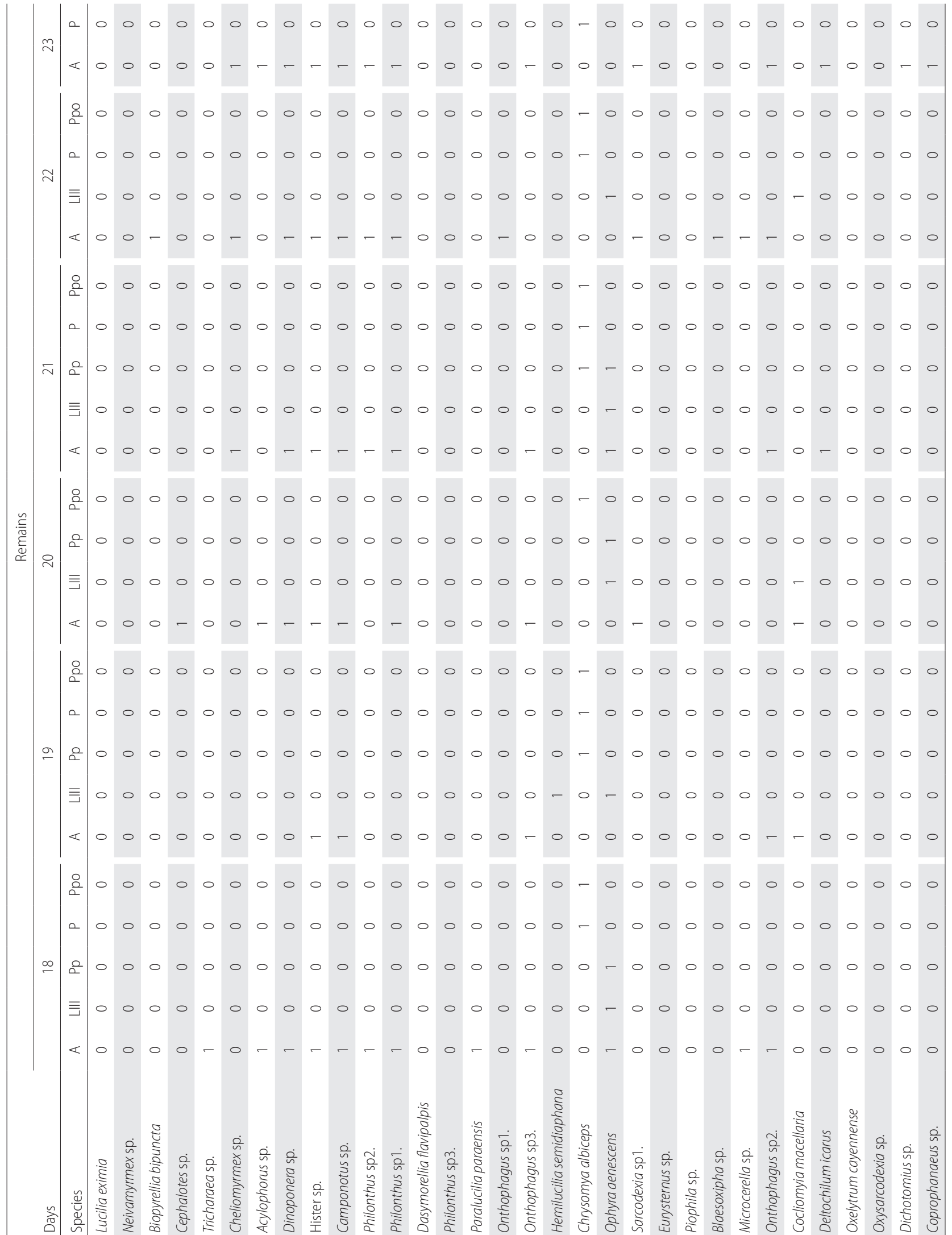

